\title{
Study of essential hypomutated genes in skin melanoma
}

\author{
I. Krivosheeva ${ }^{1}$, A. Filatova ${ }^{1}$, M. Pyatnitskiy ${ }^{2,3}$, S. Moshkovskii ${ }^{2,4}$, M. Skoblov ${ }^{1,5,6 *}$ \\ ${ }^{1}$ Research Centre of Medical Genetics, Moscow, Russia \\ ${ }^{2}$ nstitute of Biomedical Chemistry, Moscow, Russia \\ ${ }^{3}$ Higher School of Economics, Moscow, Russia \\ ${ }^{4}$ Pirogov Russian National Research Medical University, Moscow, Russia \\ ${ }_{5}^{5}$ Department of Biological and Medical Physics, Moscow Institute of Physics and Technology \\ (State University), Dolgoprudny, Russia \\ ${ }^{6}$ Far Eastern Federal University, Vladivostok, Russia \\ *e-mail:mskoblov@gmail.com
}

Key words: medical genomics, cancer, melanoma, knockdown

Motivation and Aim: Current methods of studying tumors are aimed at finding new protooncogenes, tumor suppressors, markers of cancer, etc. Information about the origination of the tumor and its genetic causes may be useful in the diagnostics, but its use for cancer therapy is limited. The searching for the genes essential for cancer cell surviving could help to develop effective cancer therapy.

Methods: For in vitro analysis we used melanoma cell line A375. We performed knockdown (KD) by lipofection with siRNAs, assessed the efficiency of KD by RTqPCR. Cell proliferation status was analyzed by MTT-test and flow cytometry. Induction of apoptosis was measured by Caspase-Glo ${ }^{\circledR}$ 3/7 Assay System. Cell migration was assessed by wound-healing assay.

Results: Using the principle named after Abraham Wald our colleagues conducted a bioinformatic analysis of mutations in the genome of the melanoma tumors and identified 91 genes not affected by considerable mutations (1). 12 out of 91 genes were selected for further analysis. We analyzed the expression level of these genes in melanoma cell line A375 by RT-qPCR. For three genes with highest expression level (UNC45A, RHPN2 and $Z N F x 1$ ) we performed knockdown (KD) experiments on the A375 cell line followed by proliferation, apoptosis and migration assays. We achieved KD efficiency for the UNC45A gene about $70 \%$, for RHPN2 - $55 \%$, and for ZNFx1 - 60\%. Caspase activity raised up to $150 \%, 125 \%$ and $200 \%$ comparing to control, respectively. Whereas proliferation assay did not reveal significant changes. Data of the migration of the cells is in progress.

Conclusion: Our results demonstrate that knockdown of chosen genes do affect caspase activity of the melanoma cells, but do not cause changes in viability of the cells. Further investigations are needed to fully confirm these results.

\section{References}

1. Pyatnitskiy M., Karpov D., Poverennaya E., Lisitsa A., Moshkovskii S. (2015) Bringing down cancer aircraft: searching for essential hypomutated proteins in skin melanoma. Chammas R., ed. PLoS ONE. 10(11):e0142819. DOI 10.1371/journal.pone.0142819. 